\title{
Prospectiva estratégica: más allá del plan estratégico
}

\begin{abstract}
RESUMEN
El presente artículo enfoca el estudio de diversas técnicas de análisis de escenarios a largo plazo, respondiendo a la siguiente interrogante ¿cómo concatenar la formulación de los diversos planes estratégicos de una organización hacia un futuro deseado en el largo plazo?; para esto se analizan y comparan tres herramientas principales tales como el Forecasting, el Foresight y la Prospectiva Estratégica, proponiéndose el esquema de la "Escalera Estratégica" como unión de los diversos planes estratégicos y el escenario futuro.
\end{abstract}

Palabras clave: Escenario futurible, prospectiva, plan estratégico, organización.

STRATEGIC PROSPECTIVE: BEYOND OF THE STRATEGIC PLAN

\section{ABSTRACT}

This article focuses its study in diverse analyze techniques of the long term scenarios, all of this answering to the next question how to link the formulation of the strategic plans of one organization to a long term desired future?; for this it analyze and compare three principal tools like the Forecasting, Foresight and the Strategic Prospective, proposing the Strategic Ladder Diagram like the link of the strategic plans and the future scenario.

Keywords: Future scenario, prospective, strategic plan, organization.

\section{INTRODUCCIÓN}

El estudio del futuro mediante la prospectiva es un tema de actualidad en la cual muchas organizaciones se preparan para afrontar el futuro que viene o para construir el suyo propio. El objetivo del presente artículo es presentar una de las principales limitaciones del plan estratégico y de cómo se puede complementar con la prospectiva estratégica.

Asimismo, otro objetivo importante es el de comparar las diversas corrientes del estudio del futuro, en este caso se ha tomado en cuenta la prospectiva estratégica, foresigth y forecasting.

El presente artículo se justifica debido a que proporcionará los lineamientos generales para la aplicación de una herramienta muy importante que podrá guiar la elaboración de los diversos planes estratégicos necesarios en el largo plazo; esto sin reemplazar la opinión de la comunidad integrante de la organización en su elaboración.

Como antecedentes de aplicaciones prácticas de la prospectiva estratégica y que han servido como base para la elaboración del presente artículo, se pueden mencionar los estudios prospectivos hechos en el Cluster de Muebles del distrito de Villa El Salvador y en el Sector Textil del distrito de San Juan de Lurigancho, ambos artículos publicados en esta misma revista (Industrial Data, Vol. 9, pp. 50-58).

\section{UNA LIMITACIÓN DEL PLAN ESTRATÉGICO}

En las diversas organizaciones e instituciones que buscan orientar su futuro, es práctica común la utilización del Plan Estratégico como herramienta clave para el desarrollo de sus actividades; sin embargo, una vez finalizado el periodo de duración de este plan queda una interrogante lógica ¿cuál debe ser el siguiente de la organización? Para responder esta interrogante existen diversas técnicas de análisis, partiendo de lo logrado hasta esa fecha en el plan estratégico que ha terminado.

Sin embargo, debido a este vacío existente muchas veces no se hace una elección adecuada o mejor dicho una elección conexa de la nueva meta de la organización, quedando muchas veces

Magíster en Ingeniería Industrial. Ingeniero Industrial. Profesor del Departamento de Diseño y Tecnología Industrial de la FII de la UNMSM. Email achungp@unmsm.edu.pe 
FIGURA 1: ESQUEMA DE LA ESCALERA PROSPECTIVA

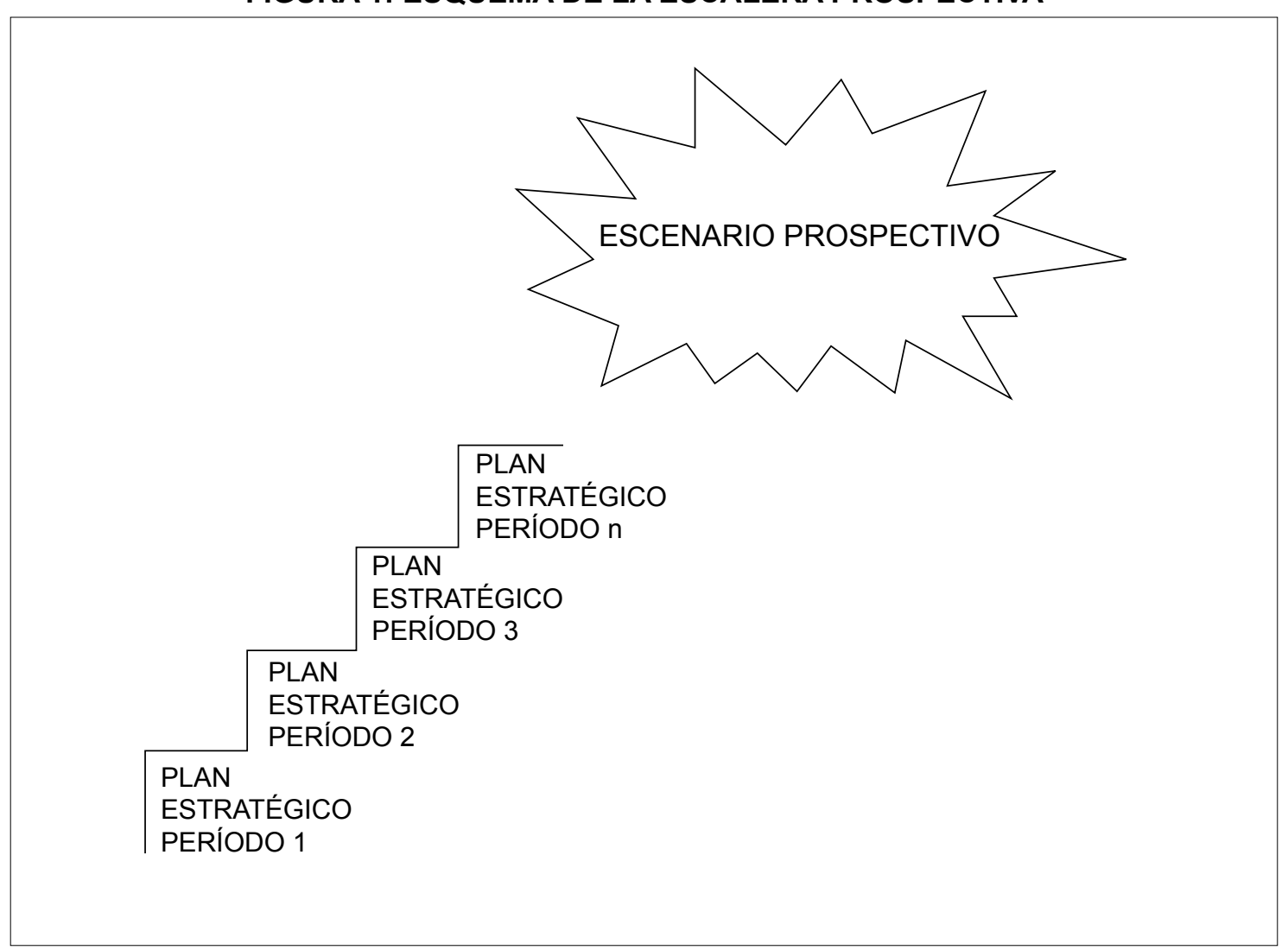

Elaboración propia.

(sobre todo en instituciones en donde se eligen a las autoridades) como un plan que recoge las aspiraciones, la línea política y sobre todo la ideología que estas personas ostentan.

Debido a lo anteriormente planteado y considerando que el plan estratégico es una guía para la organización, ¿no sería conveniente también contar con una guía complementaria en la elaboración de la visión del plan estratégico siguiente? En efecto, lo que se precisa es una orientación de hacia dónde va la organización en el largo plazo, qué futuro se desea construir, para evitar así el libre albedrío en la elaboración de este documento importantísimo, lo cual muchas veces puede degenerar en visiones inconexas, en cambio de rumbo bruscos, en otras palabras, hacia un futuro incierto.

Esta orientación no debe reemplazar de ninguna manera a la opinión de los actores involucrados en el desarrollo de la organización estudiada sino que debe orientar la opinión de estas personas y a la vez debe ser flexible en el caso que la situación lo requiera.

De lo dicho anteriormente, se puede llegar a una relación tipo "Escalera Prospectiva" en donde cada peldaño representa un plan estratégico y cada vez que éste se realiza completamente se sube una grada más hasta llegar al piso final, el cual está representado por el escenario estratégico prospectivo que, a fin de cuentas, es el piso al cual la organización desea llegar; la Figura 1 muestra gráficamente este concepto.

Conforme lo anteriormente mostrado, se puede agregar un ítem más a los documentos principales de gestión, es decir y para asentar mejor la idea, la gestión ya no empezaría con el plan estratégico sino que habría un documento superior a este (un meta documento) el cual sería la estrategia prospectiva; la Figura 2 ilustra en forma resumida el tema.

Cómo se puede observar, tanto en la Figura 2 como en la Figura 1, si una organización pudiera contar con una guía relacionada con la prospectiva, y siguiendo los pasos adecuados para la elaboración de un plan estratégico tomando como marco el escenario prospectivo, sería mucho más factible para dicha organización elegir, planificar y lograr el futuro deseado. 
FIGURA 2: NIVELES DE PLANIFICACIÓN

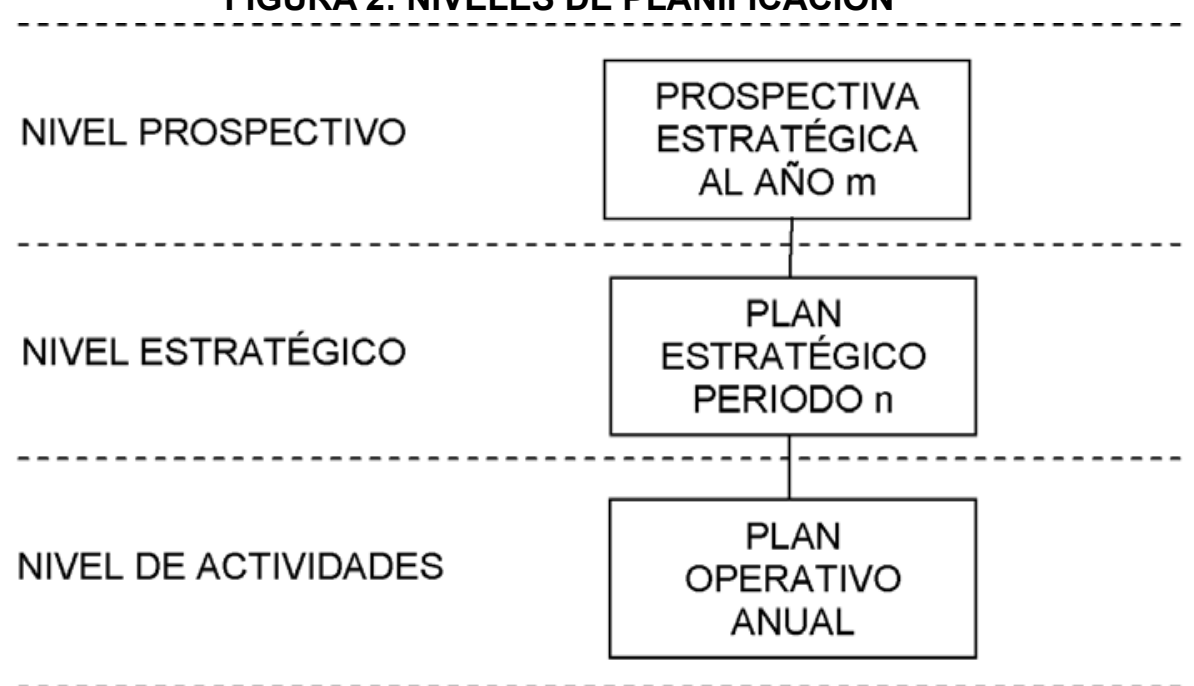

Elaboración propia.

\section{DOS CONCEPCIONES DEL FUTURO}

A continuación y para entender mejor el tema se analizarán brevemente dos formas de concebir el futuro para una organización: El Forecasting y el Foresight.

El Forecasting fue la primera herramienta para pronosticar el futuro, básicamente trabajan con otras herramientas matemáticas y estadísticas como por ejemplo: Medias móviles, regresión simple y múltiple, alisados etc., según su esquema de pensamiento el futuro es único y está condicionado a la evolución estadística de las tendencias.

En la mayoría de materias universitarias relacionadas con el tema, la enseñanza de estos métodos estadísticos se hace bajo el pensamiento Forecasting, lo cual puede constituirse en una buena forma de aproximación a esta ciencia, sin embargo sólo en un principio, ya que como se verá más adelante existen otras formas de pensar el futuro las cuales en muchos casos superarán al Forecasting.

El Foresight o la escuela anglosajona constituye una evolución del Forecasting, si bien conserva gran parte de sus principios, es decir, el análisis del futuro se basa en evolución de las tendencias, estas tendencias son analizadas en función de la opinión de expertos, por lo tanto la selección de los expertos es vital en este tipo de herramienta.

Cabe mencionar que como experto se puede seleccionar a un analista interno o externo del sistema estudiado, asimismo también puede ser un trabajador o un empresario que conozca el quehacer diario en una determinada realidad, es decir, no siempre será un erudito en la materia, puede ser una persona que se desenvuelva a diario en la organización a estudiar.

Por otro lado, el Foresight propone que el futuro si bien está condicionado por la evolución de las tendencias, las cuales se analizan según la opinión de expertos, uno puede prepararse para este futuro tomando las medidas pertinentes para afrontarlo.

Entre sus principales herramientas de análisis se tienen: Delphi, construcción de escenarios, seminarios de trabajos estructurados, análisis de escenarios y análisis de impactos.

\section{UNA NUEVA CONCEPCIÓN DEL FUTURO: LA PROSPECTIVA ESTRATÉGICA}

Finalmente, la tercera técnica a analizar es la Prospectiva Estratégica, también conocida como la escuela francesa, la cual fue impulsada por el Dr. Michael Godet, miembro de LIPSOR. En este caso se propone un rompimiento con el pensamiento de los métodos anteriores básicamente en dos aspectos:

El futuro no depende de la evolución de las tendencias sino de la relación que existe entre los factores del sistema, actores y objetivo. En efecto bajo esta perspectiva el futuro con los posibles escenarios futuribles se darán en función a cómo interactúan los agentes mencionados anteriormente, esto no deja de lado la importancia de las tendencias, pero indica que la relación entre los agentes tales como factores del sistema, actores y objetivo condicionará su comportamiento y por lo tanto el desarrollo de los eventos. 


\section{CUADRO RESUMEN DE LOS TRES MÉTODOS ESTUDIADOS}

\begin{tabular}{|c|c|c|}
\hline FORECASTING & FORESIGHT & PROSPECTIVA ESTRATÉGICA \\
\hline 1. Se basa tendencias. & 1. Se basa en tendencias. & $\begin{array}{l}\text { 1. Se basa en las relaciones de las } \\
\text { variables, actores y objetivos. }\end{array}$ \\
\hline $\begin{array}{l}\text { 2. Estudia su evolución mediante } \\
\text { herramientas estadísticas. }\end{array}$ & $\begin{array}{l}\text { 2. Estudia su evolución por la } \\
\text { opinión de expertos. }\end{array}$ & $\begin{array}{l}\text { 2. Estudia sus relaciones mediante } \\
\text { opiniones cualitativas que las } \\
\text { transforma en cuantitativas. }\end{array}$ \\
\hline 3. El futuro es uno e inevitable. & $\begin{array}{l}\text { 3. El futuro es uno, debemos } \\
\text { prepararnos para él. }\end{array}$ & $\begin{array}{l}\text { 3. No hay un solo futuro, existen } \\
\text { varios escenarios futuribles, uno } \\
\text { busca su futuro. }\end{array}$ \\
\hline $\begin{array}{l}\text { 4. El futuro está condicionado por } \\
\text { la evolución estadística. }\end{array}$ & $\begin{array}{l}\text { 4. El futuro se analiza con la } \\
\text { opinión de expertos. }\end{array}$ & $\begin{array}{l}\text { 4. Los escenarios futuribles se } \\
\text { determinan por relaciones. }\end{array}$ \\
\hline
\end{tabular}

Elaboración propia.

No existe un solo futuro sino varios escenarios futuribles posibles, debido a lo mencionado en el punto anterior, se puede concluir que el comportamiento de los agentes que determinan el futuro del sistema puede ser variado, por lo tanto, no existe un único futuro sino varios futuros, lo que en prospectiva estratégica se conoce como "escenarios futuribles", lo cual representa una gran evolución con respecto a las técnicas estudiadas anteriormente. En otras palabras, lo que se busca con la prospectiva estratégica no es pronosticar un futuro y está resignado a él, o prepararse para afrontar ese futuro; al contrario la esencia de la prospectiva estratégica consiste en construir el futuro deseado lo que se conoce como escenario apuesta.

Trabajar con Prospectiva Estratégica implica también el trabajo con expertos, convirtiendo lo cualitativo en cuantitativo mediante sus herramientas de trabajo; estas herramientas se encuentran detalladas en el libro "Caja de Herramientas de la Prospectiva Estratégica" del Dr. Godet; sin embargo, el trabajo básico se puede realizar con las siguientes técnicas:

a. Análisis MICMAC, en donde se identifican los factores claves y sus relaciones mediante las matrices de impacto directo o el indirecto.

b. Análisis MACTOR, en donde se identifican los principales actores, objetivos del sistema, la relación entre actores $\mathrm{x}$ actores $\mathrm{y}$ actores $\mathrm{x}$ objetivos.

c. Análisis de escenarios, en donde se identifican las principales hipótesis según los factores claves, según ellos se construye en los principales escenarios futuribles y luego se analizan en forma probabilística en su ocurrencia, seleccionando el escenario a pues.
El software disponible para realizar el trabajo de prospectiva, se puede descargar gratuitamente en la siguiente dirección:

http://www.3ie.fr/lipsor/lipsor_es/index_es.htm

\section{RESUMIENDO LOS TRES MÉTODOS ESTUDIADOS}

En el cuadro resumen adjunto se presenta las características de los tres métodos mencionados.

A pesar de las diferencias mencionadas, los tres métodos no se excluyen el uno del otro, en muchos casos pueden complementarse, como ejemplo de esto se puede mencionar el estudio del Futuro al 2020 de la Educación Superior realizado por el Convenio Andrés Bello, sin embargo, la filosofía del estudio que se vaya realizar en caso de combinar los métodos debería ajustarse a una sola concepción acerca de cómo debe ser el futuro o los futuros.

\section{CONCLUSIONES}

1. El plan estratégico resulta insuficiente en una organización que desee proyectarse al largo plazo.

2- El esquema de la "Escalera Estratégica" es la relación adecuada entre Plan Estratégico y Prospectiva Estratégica.

3. No existe una sola concepción acerca del futuro, sino que esta concepción depende del método de estudio que se utilice.

4. Se pueden combinar los métodos de estudio del futuro, pero deben de estar regidos bajo una sola concepción de dicho futuro.

5. El escenario futurible debe constituirse como una guía o norte para la elaboración de los Planes Estratégicos. 


\section{REFERENCIAS BIBLIOGRÁFICAS}

Convenio Andrés Bello (2005). Documentos del Estudio de la Educación Superior al 2020. Convenio Andrés Bello.

EULAKS (2009). Boletín 1. EULAKS.

Godet M. (2000). Caja de herramientas de la prospectiva estratégica. 4ta. edición. Instituto Europeo de Prospectiva Estratégica. España.

Inche J., Chung A. (2006). Prospectiva estratégica en el cluster de muebles de Villa el Salvador. Industrial Data Vol. 9.
Keenan M., Scapolo F. (2004). Pilot Project to Scope the Establishment of a European Foresigth Academy. Institute for Prospective Technological Studies.

López A. (2009). Prospectiva y cambio social. ARBOR 825-836.

Medina J. (2007). Manual de prospectiva y decisión. ONU. Chile.

Mojica F. (2006). Concepto y aplicación de la prospectiva estratégica. Med 14(1):122-131.

Pricewaterhousecooper. Manual de consulta Forecasting. Pricewaterhousecooper. 